\title{
Epithelial-myoepithelial carcinoma of the lung: a case report
}

Yasuhiro Nakashima ${ }^{1,3^{*}}$, Riichiro Morita ${ }^{1}$, Akiko Ui ${ }^{1,3}$, Kuniko lihara $^{2}$ and Takuya Yazawa ${ }^{4}$

\begin{abstract}
Background: Pulmonary epithelial-myoepithelial carcinoma (P-EMC) is a rare subset of salivary gland-type tumors of the lung. Because of its rarity and unproven malignant potential, the optimal therapy for P-EMC has not been defined. Here, we report a typical case of P-EMC and a review of the literature to consider appropriate treatment.

Case presentation: A 54-year-old woman presented with an abnormal lung shadow on a routine chest X-ray. A chest computed tomography (CT) scan verified an 18-mm endobronchial nodule on the middle lobe. We performed a bronchoscopic biopsy, and the patient was diagnosed with P-EMC. After confirming the absence of tumors in the salivary glands, she underwent a right middle lobectomy along with hilar and mediastinal lymph node dissections. Currently, the patient is doing well, without any sign of recurrence 3 years after surgery.

Conclusions: Although a majority of P-EMC cases, as in our case, behave indolently, several poor progression cases have been reported. For distinguishing the minor malignancy cases from others, histological findings such as myoepithelial anaplasia could be a predictive factor. Complete resection is needed to evaluate the whole tumor, because P-EMCs often show histological heterogeneity. Moreover, incomplete excision may be a poor prognostic factor. Although lobectomies as well as lymph node dissections, sleeve lobectomies, or pneumonectomies are routinely performed for complete resection, further investigation is required to establish the optimal treatment strategy.
\end{abstract}

Keywords: Pulmonary epithelial-myoepithelial carcinoma (P-EMC), Epithelial-myoepithelial tumor, Pulmonary salivary glandtype tumor, Lung cancer

\section{Background}

Pulmonary epithelial-myoepithelial carcinoma (P-EMC) is a rare subset of salivary gland-type tumors of the lung. Although it is generally regarded as a low-grade malignant tumor and typically behaves indolently [1], distant metastases and recurrences occasionally occur. Some pathologists describe the malignant potential of P-EMC as "unproven," rather than "low-grade malignant" [2]. Because of its rarity and unproven malignant potential, optimal therapy for P-EMC has not been defined. Here we report a typical case of P-EMC and a review of the literature to consider appropriate treatment.

\footnotetext{
* Correspondence: midysland@gmail.com

The subject matter of this case report was presented at the 32nd Annual Meeting of the Japanese Association for Chest Surgery, 14-15 May 2015

${ }^{1}$ Department of Chest Surgery, Tokyo Yamate Medical Center, 3-22-1

Hyakunin-cho, Shinjuku-ku, Tokyo 169-0073, Japan

${ }^{3}$ Department of Thoracic Surgery, Tokyo Medical and Dental University,

1-5-45, Yushima, Bunkyo-ku, Tokyo 113-8519, Japan

Full list of author information is available at the end of the article
}

\section{Case presentation}

A 54-year-old female patient presented with an abnormal shadow discovered on a routine chest X-ray. She had a history of smoking 4-5 cigarettes per month for 5 years but quit over 10 years ago. Her past medical history included a colorectal benign polyp resected by endoscopy. She did not have respiratory symptoms and laboratory findings were unremarkable. The serum levels of the tumor markers (carcinoembryonic antigen, squamous cell carcinoma antigen, and cytokeratin 19 fragment) were within normal limits. A chest radiograph showed a nodular shadow at the right middle lung field (Fig. 1a), and a computed tomography (CT) scan confirmed an $18-\mathrm{mm}$ lobulated nodule at the middle lobe (Fig. 1b, c). An $\mathrm{F}^{18}$-fluoro-deoxy-glucose positron emission tomography/CT (FDG-PET/CT) scan did not indicate abnormal FDG uptake. Bronchoscopy showed the round, tan, solid endobronchial nodule reducing the lumen of the right subsegmental bronchus $\left(\mathrm{B}_{\mathrm{a}}^{5}\right.$ ) (Fig. 1d). A bronchoscopic biopsy was 


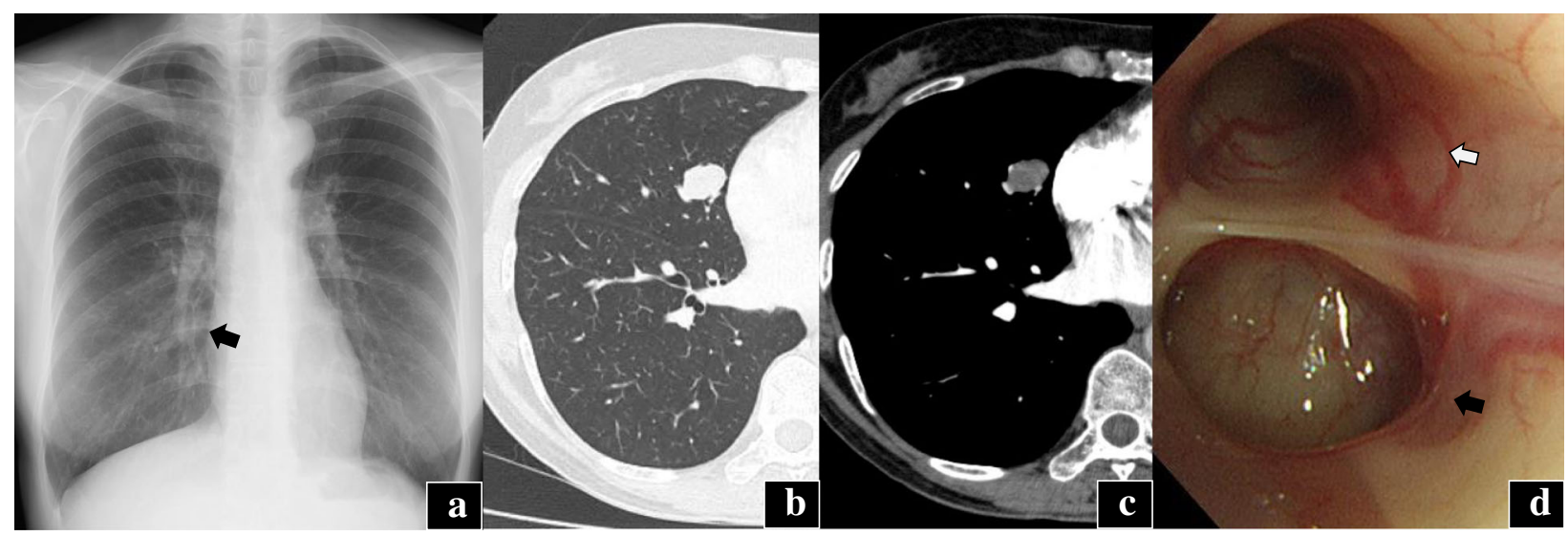

Fig. 1 Medical imaging findings of the nodule. a Chest X-ray reveals a 2-cm shadow in the right middle lung field (black arrow). b, c CT scan reveals an 18-mm lobulated nodule. $\mathbf{d}$ Bronchoscopy shows the endobronchial nodule reducing the lumen of right $\mathrm{B}^{5}$ a sub-segmental bronchus (black arrow) and the remaining patency of the $\mathrm{B}^{5} \mathrm{~b}$ sub-segmental bronchus (white arrow)

performed, and the patient was diagnosed with an epithelial-myoepithelial carcinoma (EMC). Examination of otolaryngologist and magnetic resonance imaging (MRI) of the head revealed no salivary gland pathologies. A right pulmonary middle lobectomy was performed, along with hilar and mediastinal lymph node dissections.
The tumor was measuring $15 \mathrm{~mm}$ in diameter and had a white surface; it was well-circumscribed and was present along the bronchial wall (Fig. 2a). On histological examinations, the tumor was located in the submucosal layer of the bronchus, oppressing the adjacent bronchioles, and partly necrotic (Fig. 2b, c). The tumor consisted of two different components: the duct-forming

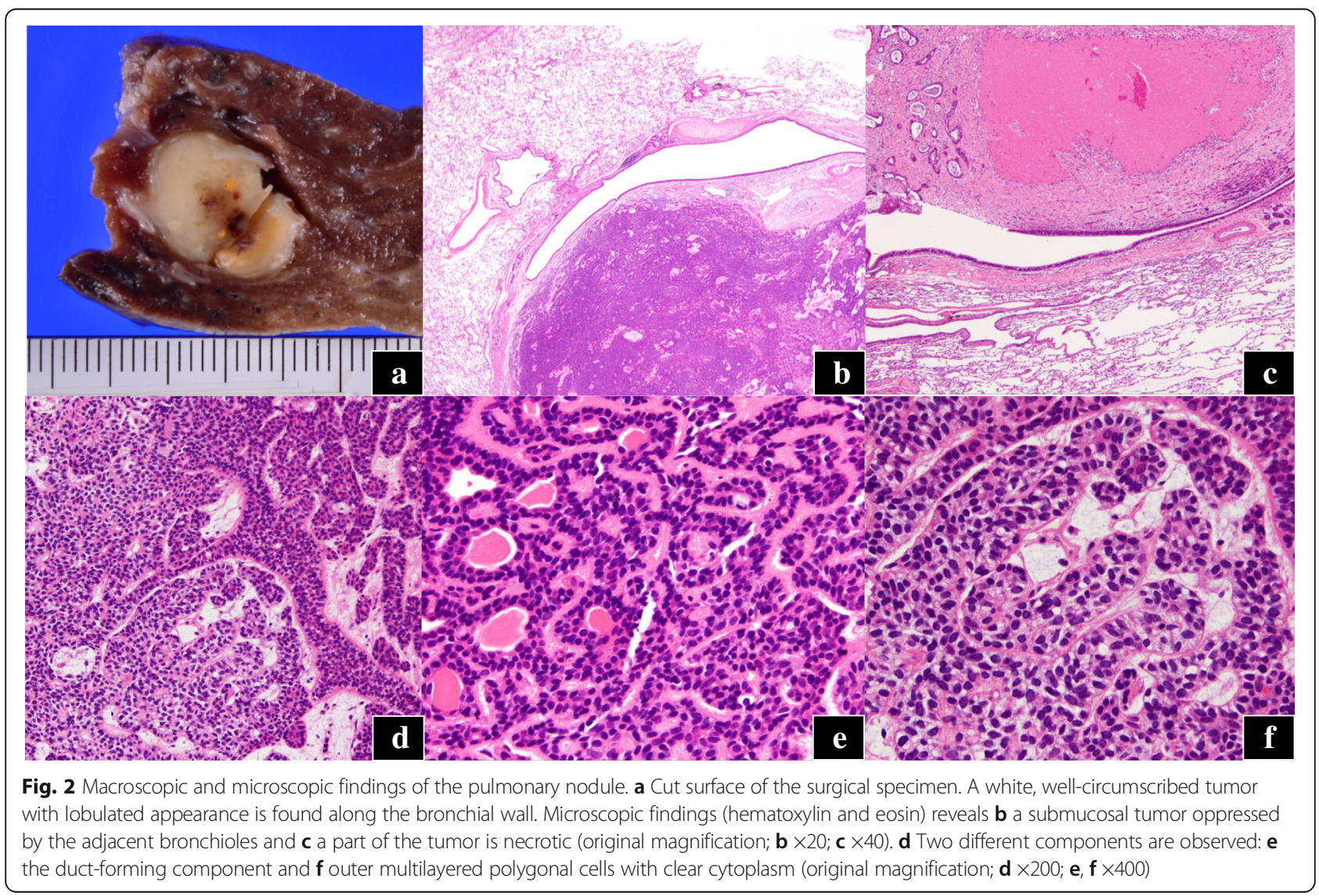


epithelial cells and outer multilayered polygonal cells with clear cytoplasm (Fig. 2d-f). Duct-forming epithelial cells were positive for cytokeratin 7 , while the outer cells were negative (Fig. 3a). The outer cells were positive for S100 protein, smooth muscle actin (SMA), p63, and cytokeratin 5/6 (Fig. 3b-d), suggesting myoepithelial phenotype. Neither vascular, lymphatic, nor neural invasion was observed, and the mitotic rate is rare. The Ki-67 labeling index was less than 5\% (Fig. 3e). No mutation was found in the KRAS or EGFR gene. We finally diagnosed the patient with P-EMC. The patient is doing well without any sign of recurrence 3 years after surgery.

\section{Discussion}

P-EMC is a tumor characterized by biphasic morphology, consisting of an inner layer of duct-like structures made of epithelial cells and a surrounding layer of myoepithelial cells immunoreactive for S-100 and smooth muscle actin [1]. Precise diagnosis of this tumor via preoperative bronchoscopy is difficult because of its revealing heterogeneity; however, biphasic features in our biopsy specimen allowed us to make the diagnosis of P-EMC before surgery.

Even when a preoperative diagnosis of P-EMC is possible, optimal treatment methods and follow-up periods have not been established due to the tumor's unproven malignant potential [2]. Our review of the literature revealed a total of 56 P-EMC cases, including our case, in the English literature (Tables 1 and 2) [2-27]. We found reported cases of 32 females and 24 males, with an average age of 56 years (age range, 7-81 years). Forty-five cases had tumors localized in the central airway within segmental bronchi and appeared to be endobronchial masses. On the contrary, 11 cases had tumors localized in the pulmonary parenchyma $[3,14,18,23,27]$, and 5 of these cases were reported with tumors clearly presenting as intraparenchymatous masses without apparent connection with a bronchus [3, 18, 27]. Because endobronchial localizations and the histologic features mimic those of salivary gland tumors, P-EMCs are regarded as originating from the epithelium of submucosal bronchial glands. However, the existence of these tumors in the peripheral lung tissue suggests that P-EMC might originate from primitive cells [3]. In the 25 cases we reviewed, patients presented with symptoms of bronchial obstruction such as productive cough, fever, and dyspnea. Although our patient was asymptomatic, obstruction of her sub-segmental bronchus would have eventually caused symptoms. As one of the reasons why poor clinical courses in cases of P-EMC are fewer than those of salivary gland EMC, it is considered that obstructive bronchial symptoms often appear [26]. In many cases we reviewed, as in our case, CTs demonstrated that the masses had comparatively clear boundaries and homogeneous densities. While the most frequently reported P-EMCs do not reveal abnormal FDG uptake in FDG-PET/CT scans as in this case, three cases revealed active FDG uptake [20,23, 25], and one of those had hilar and subcarinal lymph node metastasis [25].

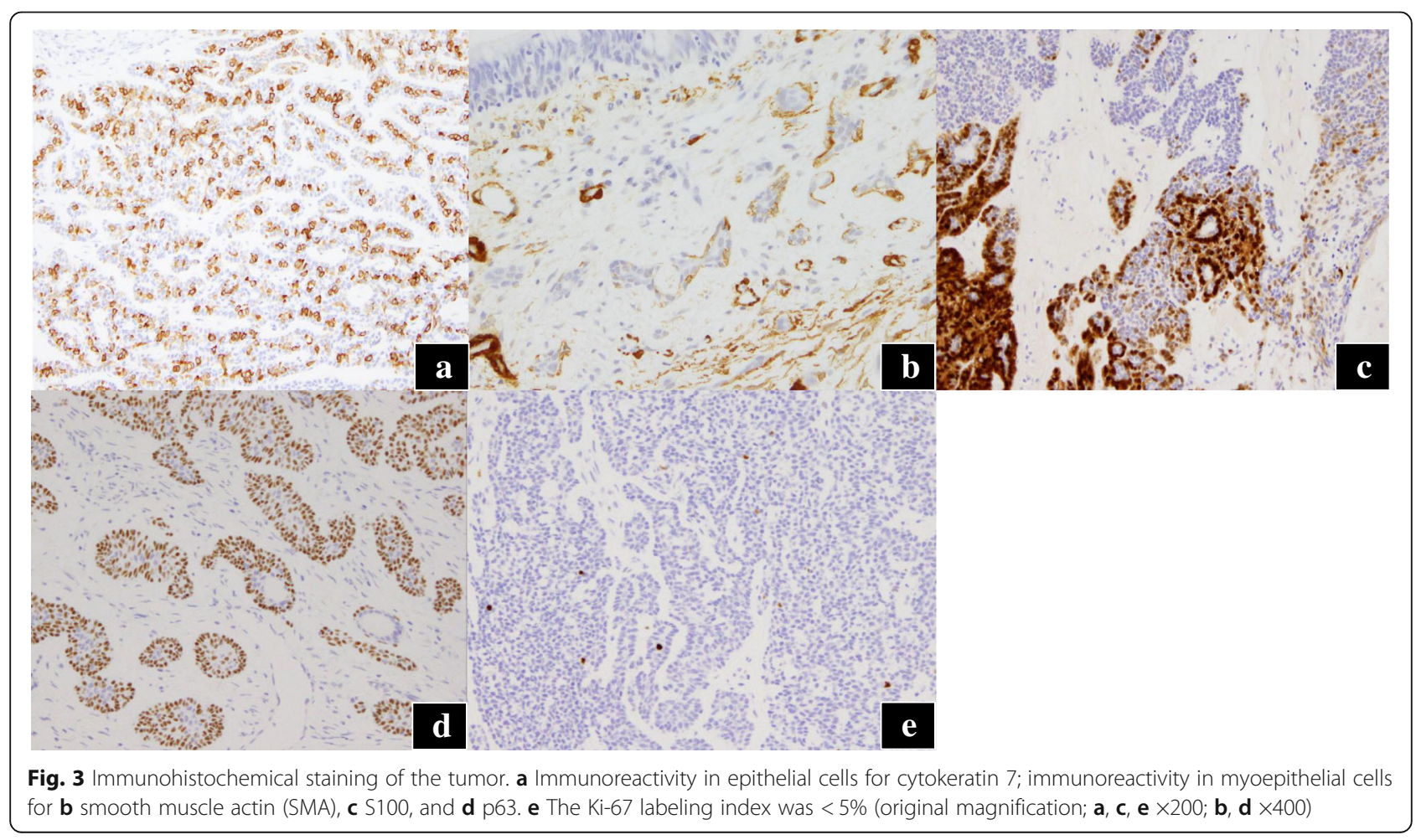


Table 1 Review of P-EMC cases: Clinical characteristics and surgical procedure

\begin{tabular}{|c|c|c|c|c|c|c|c|c|c|}
\hline Year & Author & Age & Sex & $\begin{array}{l}\text { Obstructive } \\
\text { airway symptoms }\end{array}$ & Location & $\begin{array}{l}\text { Location (endo- } \\
\text { bronchial or not) }\end{array}$ & $\begin{array}{l}\text { Surgical } \\
\text { procedure }\end{array}$ & $\begin{array}{l}\text { Mediastinal lymph } \\
\text { node dissection }\end{array}$ & Size $(\mathrm{cm})$ \\
\hline \multirow[t]{8}{*}{1994} & \multirow[t]{8}{*}{ Moran CA et al. [3] } & $1: 47$ & $\mathrm{~F}$ & + & LMB & $E$ & Pneumo & NA & 2.5 \\
\hline & & 2: 45 & $\mathrm{~F}$ & - & LLL & $P, U$ & L & NA & 2.5 \\
\hline & & 3: 42 & $\mathrm{~F}$ & - & RLL & $\mathrm{E}$ & L & NA & 2.5 \\
\hline & & 4: 57 & M & - & RUL & $P, U$ & $L$ & NA & 2.0 \\
\hline & & 5: 58 & $\mathrm{~F}$ & + & LUL & $\mathrm{E}$ & L & NA & 2.0 \\
\hline & & 6: 35 & $\mathrm{~F}$ & + & RLL & $\mathrm{E}$ & L & NA & 16.0 \\
\hline & & $7: 67$ & M & + & RUL & $E$ & $L$ & NA & 6.0 \\
\hline & & $8: 69$ & $\mathrm{~F}$ & - & LLL & $P, U$ & L & NA & 2.0 \\
\hline 1994 & Nistal et al. [4] & 55 & $\mathrm{~F}$ & + & RULB & $\mathrm{E}$ & L & NA & 2.0 \\
\hline 1995 & Tsuji et al. [5] & 66 & M & NA & $\mathrm{RMB}$ & $\mathrm{E}$ & Pneumo & NA & 16 \\
\hline 1997 & Wilson RW et al. [6] & 55 & $\mathrm{~F}$ & + & LLSB & $\mathrm{E}$ & L & NA & 3.9 \\
\hline 1998 & Shanks et al. [7] & 67 & M & + & LLLB & $\mathrm{E}$ & L & NA & 1.3 \\
\hline 1998 & Ryska et al. [8] & 47 & $\mathrm{~F}$ & + & RULB & $\mathrm{E}$ & B & - & NA \\
\hline \multirow[t]{4}{*}{2001} & \multirow[t]{4}{*}{ Fulford LG et al. [9] } & 1: 55 & $\mathrm{~F}$ & + & RMB & $\mathrm{E}$ & Pneumo & NA & 5.0 \\
\hline & & 3: 56 & M & + & $\begin{array}{l}\text { Lobe } \\
\text { bronchus side } \\
\text { unstated }\end{array}$ & $E$ & $L$ & NA & NA \\
\hline & & 4: 57 & $\mathrm{~F}$ & + & LMB & $\mathrm{E}$ & Pneumo & NA & 1.5 \\
\hline & & 5: 54 & $\mathrm{~F}$ & NA & RULB & $\mathrm{E}$ & $L$ & NA & 1.5 \\
\hline 2001 & Pelosi et al. [2] & 47 & M & & LULB & $\mathrm{E}$ & SL & + & 1.5 \\
\hline 2003 & Doganay et al. [10] & 73 & M & + & LLLB & $\mathrm{E}$ & Pneumo & + & 5 \\
\hline 2004 & Ru et al. [11] & 73 & M & + & LULB & $E$ & $L$ & - & 3.8 \\
\hline 2007 & Chao et al. [12] & 43 & $\mathrm{~F}$ & + & LMB & $\mathrm{E}$ & B & - & NA \\
\hline 2007 & Musulimani et al. [13] & 74 & M & + & LMB & $\mathrm{E}$ & $\mathrm{B}$ & - & NA \\
\hline \multirow[t]{5}{*}{2007} & \multirow[t]{5}{*}{ Chang et al. [14] } & 1: 54 & $\mathrm{~F}$ & - & RLL & $P_{,}^{*} 1$ & W & - & 2.6 \\
\hline & & $2: 62$ & $\mathrm{~F}$ & - & LLL & $P, * 1$ & W & - & 2 \\
\hline & & 3: 58 & $\mathrm{~F}$ & + & RML & $P,{ }^{*} 1$ & W & - & 1.2 \\
\hline & & 4: 57 & $\mathrm{~F}$ & - & LUL & $P, * 1$ & W & - & 0.8 \\
\hline & & 5: 52 & $\mathrm{~F}$ & - & $\begin{array}{l}\text { RUL(bilateral } \\
\text { nodules) }\end{array}$ & $P,{ }^{* 1}$ & W & - & 1.2 \\
\hline \multirow[t]{5}{*}{2009} & \multirow[t]{5}{*}{ Nguen et al. [15] } & 1: 38 & M & - & LLL & $\mathrm{E}$ & L & - & 5 \\
\hline & & 2: 48 & M & - & RUL & $E$ & L & - & 2.5 \\
\hline & & 3: 52 & $\mathrm{~F}$ & - & LLL & $\mathrm{E}$ & L & - & 3 \\
\hline & & 4: 54 & M & - & RUL & $\mathrm{E}$ & $L$ & - & 3 \\
\hline & & 5: 56 & $\mathrm{~F}$ & - & LMB & $E$ & Pneumo & NA & 4.2 \\
\hline 2009 & Rosenfeld et al. [16] & 7 & M & - & RLSB & $\mathrm{E}$ & L & N/A & 3.6 \\
\hline 2011 & Nishihara et al. [17] & 81 & M & - & RULB & $\mathrm{E}$ & $\begin{array}{l}\text { Biopsy only, } \\
\text { BSC }\end{array}$ & - & NA \\
\hline 2011 & Munoz et al. [18] & 76 & $\mathrm{~F}$ & - & RUL & $P, U$ & $L$ & NA & 2.7 \\
\hline 2011 & Kang et al. [19] & $\begin{array}{l}\text { 1-2: } \\
\text { median } 57.0\end{array}$ & $\begin{array}{l}M(1) \\
F(1)\end{array}$ & NA & $\begin{array}{l}\operatorname{LUL}(1) \\
\operatorname{LLL}(1)\end{array}$ & NA & $\begin{array}{l}\text { SL(1) } \\
\text { Pneumo(1) }\end{array}$ & $+(2)$ & Median 6.9 \\
\hline 2012 & Arif et al. [20] & 57 & M & - & $\begin{array}{l}\text { Rt. } \\
\text { Intermedius } \\
\text { bronchus }\end{array}$ & $\mathrm{E}$ & Bi-L & NA & 1.2 \\
\hline 2013 & Zhu et al. [21] & $\begin{array}{l}\text { 1 7: } \\
\text { median } 63 \\
(36-75)\end{array}$ & $\begin{array}{l}M(3) \\
F(4)\end{array}$ & $+(3)$ & $\begin{array}{l}\operatorname{RMB}(2) \\
\operatorname{RUL}(1) \\
\operatorname{RLL}(2)\end{array}$ & NA & $\begin{array}{l}\mathrm{L}(5) \\
\mathrm{SL}(1) \\
\text { Pneumo(1) }\end{array}$ & NA & $\begin{array}{l}\text { Median } 2.5 \\
(1.3-4.0)\end{array}$ \\
\hline
\end{tabular}


Table 1 Review of P-EMC cases: Clinical characteristics and surgical procedure (Continued)

\begin{tabular}{|c|c|c|c|c|c|c|c|c|c|}
\hline Year & Author & Age & Sex & $\begin{array}{l}\text { Obstructive } \\
\text { airway symptoms }\end{array}$ & Location & $\begin{array}{l}\text { Location (endo- } \\
\text { bronchial or not) }\end{array}$ & $\begin{array}{l}\text { Surgical } \\
\text { procedure }\end{array}$ & $\begin{array}{l}\text { Mediastinal lymph } \\
\text { node dissection }\end{array}$ & Size $(\mathrm{cm})$ \\
\hline & & & & & $\begin{array}{l}\operatorname{LUL}(1) \\
\operatorname{LLL}(1)\end{array}$ & & & & \\
\hline 2013 & Konoglou et al. [22] & 34 & M & + & Trachea & $E$ & $\begin{array}{l}\text { Resection of } \\
\text { five tracheal } \\
\text { rings }\end{array}$ & - & 1.15 \\
\hline 2014 & Cho et al. [23] & 51 & $\mathrm{~F}$ & - & LUL & $P, N A$ & L & + & 3.3 \\
\hline \multirow[t]{5}{*}{2014} & Song et al. [24] & $1: 52$ & $\mathrm{~F}$ & + & LLL & $E$ & L & NA & 12 \\
\hline & & $2: 66$ & M & + & LUL & $E$ & SL & NA & 1.8 \\
\hline & & 3: 60 & M & - & LUL & $E$ & L & NA & 0.7 \\
\hline & & 4: 61 & M & + & RUL & E & L & NA & 1.5 \\
\hline & & $5: 63$ & $\mathrm{~F}$ & + & Trachea & E & B & - & 2 \\
\hline 2015 & Cha et al. [25] & 53 & $\mathrm{~F}$ & + & $\begin{array}{l}\text { Rt. } \\
\text { intermedius } \\
\text { bronchus }\end{array}$ & E & Bi-L(VATS) & + & 2.2 \\
\hline 2015 & Tajima et al. [26] & 72 & $\mathrm{~F}$ & - & LBSB & $E$ & L(VATS) & + & 3.8 \\
\hline 2016 & Shen et al. [27] & 58 & M & - & LLL & $P, U$ & NA(VATS) & NA & 1.3 \\
\hline Curren & nt case & 54 & $\mathrm{~F}$ & - & RMLB & E & L & + & 1.5 \\
\hline
\end{tabular}

$M$ male (number of people), $F$ female (number of people), $N / A$ not available, $L M B$ left main bronchus, $L L L$ left lower lobe, $R L L$ right lower lobe, $R U L$ right upper lobe, $L U L$ left upper lobe, $R U L B$ right upper lobe bronchus, RMB right main bronchus, $L B S B$ left basal segment bronchus, $L L L B$ left lower lobe bronchus, $L U L B$ left upper lobe bronchus, $R M L$ right middle lobe, $R L S B$ right lower lobe segment bronchus, $L()$ lobe(number of people), $R t$. right, $R M L B$ right middle lobe bronchus, $E$ endobronchial, $P$ pulmonary parenchyma, $U$ unrelated to a bronchus, ${ }^{*} 1$ located in the periphery of the lung, did not involve any large bronchi, close proximity to a small caliber airway, Pneumo pneumonectomy, $L$ lobectomy, $B$ endobronchial excision, $S L$ sleeve lobectomy, $W$ wedge resection, $B S C$ best supportive care, $B i-L$ bi-lobectomy, VATS video-assisted thoracic surgery

Although P-EMC cases are typically indolent, they are potentially malignant, and recurrence and metastasis may occur. Clinical follow-up information is provided for 50 cases in this review. Six cases of recurrence and four cases of metastasis have been reported thus far, and two of the six patients with recurrence died of P-EMC. The size of the tumors varied, ranging from 0.7 to $16 \mathrm{~cm}$ in diameter, with an average of $2.5 \mathrm{~cm}$. The size of P-EMC that occurred in the metastasis or recurrence tended to be larger than the average size of P-EMC. The size of P-EMCs causing lymph node metastases or recurrence were $3.6 \mathrm{~cm} \mathrm{[16]} \mathrm{and} 2.2 \mathrm{~cm} \mathrm{[25]} \mathrm{or} 16 \mathrm{~cm} \mathrm{[3],}$ $6 \mathrm{~cm}$ [3], and $12 \mathrm{~cm}$ [24], respectively. All 11 tumors localized in pulmonary parenchyma showed no evidence of recurrence or metastasis.

There are three histological distinct subtypes of P-EMC: one presents with a dual ductal component, which is a defined characteristic feature of this tumor (19 cases including our case); one presents with a solid component mainly consisting of spindle and polygonal-shaped myoepithelial cells (14 cases); and one mainly consists of myoepithelial cells with increased nuclear atypia, called myoepithelial anaplasia (four cases) [3, 25, 26]. For distinguishing the minor malignant cases from others, many pathologists have attempted to identify a specific histopathological finding as a predictive factor. Poor prognostic factors of the salivary gland EMC are often applied to P-EMCs. Seethala et al. reported that positive margin status, presence of angiolymphatic invasion, necrosis, and myoepithelial anaplasia in the EMC in salivary glands were predictors of decreased disease-free survival (DFS). Histology of both patients who died of P-EMC showed myoepithelial cell-predominant features with anaplasia [3, 24]. The other three cases having a component of myoepithelial anaplasia showed the tumor progression: a case recurred 2 years after lobectomy [3], lymph node metastasis was found at the surgery in a case [25], and pulmonary infiltration was found in a case [26]. Therefore, myoepithelial anaplasia could be one of the predictive poor prognostic factors of P-EMC.

Complete resection is needed to evaluate the whole tumor, which usually shows histological heterogeneity. Moreover, incomplete excision may be a predictor of poor prognosis for P-EMC, as it is in salivary gland EMC [28]. Despite the fact that most of P-EMCs are indolent, various kinds of surgical procedures have been frequently performed until now for complete resections. Among the cases we reviewed, the following procedures were performed: a partial resection of the trachea (1 case), lobectomy (28 cases), sleeve lobectomy (4 cases), bi-lobectomy (2 cases), and pneumonectomy (8 cases). Other less-invasive procedures were performed in a few 
Table 2 Review of P-EMC cases: Cases and clinicopathological features

\begin{tabular}{|c|c|c|c|c|c|c|c|}
\hline Year & Author & $\begin{array}{l}\text { Predominant } \\
\text { component }\end{array}$ & $\begin{array}{l}\text { High mitotic rate/ } \\
\text { necrosis/Ly,V,N invasion }\end{array}$ & Ki-67 & p53 & Metastasis & F/U (months) \\
\hline \multirow[t]{8}{*}{1994} & \multirow[t]{8}{*}{ Moran CA et al. [3] } & G & $-/-/-$ & NA & NA & Free & $\mathrm{FOD}(72)$ \\
\hline & & M & $(2-3 / 10 \mathrm{HPF}) /+/-$ & NA & NA & Free & $\mathrm{FOD}(48)$ \\
\hline & & M & $-/-1-$ & NA & NA & Free & NA \\
\hline & & M & $(2-3 / 10 \mathrm{HPF}) /+/-$ & NA & NA & Free & Died of surgery(0) \\
\hline & & G & $-/-/-$ & NA & NA & Free & NA \\
\hline & & M & (5-10/10HPF)/+/- & NA & NA & Free & Recurred LN mets after 2 years \\
\hline & & M & $(5-10 / 10 \mathrm{HPF}) /+/+$ & NA & NA & Free & $\begin{array}{l}\text { Recurred after } 3 \text { years in } \\
\text { trachea } \\
\text { After CRTx, mets to multiple } \\
\text { organs } \\
\text { Died of P-EMC. }\end{array}$ \\
\hline & & M & $(2-3 / 10 \mathrm{HPF}) /-/-$ & NA & NA & Free & NA \\
\hline 1994 & Nistal et al. [4] & G & Scanty/NA/- & NA & NA & Free & $\mathrm{FOD}(24)$ \\
\hline 1995 & Tsuji et al. [5] & M & Rarely/+/- & NA & NA & Free & $\begin{array}{l}\text { FOD(36), died of unrelated } \\
\text { disease. }\end{array}$ \\
\hline 1997 & Wilson RW et al. [6] & G & $-/-/-$ & NA & NA & Free & $\mathrm{FOD}(7)$ \\
\hline 1998 & Shanks et al. [7] & G & $(1 / 20 \mathrm{HPF}) /-/-$ & NA & NA & Free & NA \\
\hline 1998 & Ryska et al. [8] & G & $\mathrm{NA} / \mathrm{NA} / \mathrm{NA}$ & NA & NA & Free & $\mathrm{FOD}(13)$ \\
\hline \multirow[t]{4}{*}{2001} & \multirow[t]{4}{*}{ Fulford LG et al. [9] } & G & $(1 / 20 \mathrm{HPF}) /-/-$ & $2-10 \%$ & NA & Free & $\mathrm{FOD}(8)$ \\
\hline & & G & $(1 / 20 \mathrm{HPF}) /-/-$ & $1-2 \%$ & NA & Free & $\mathrm{FOD}(60)$ \\
\hline & & M & $-/-1-$ & $<1 \%$ & NA & Free & FOD(96) \\
\hline & & M & $(1 / 20 \mathrm{HPF}) /+/-$ & $1-2 \%$ & NA & Free & $\mathrm{FOD}(84)$ \\
\hline 2001 & Pelosi et al. [2] & NA & $-1-1-$ & $\begin{array}{l}\text { G) } 1.5 \% \\
\text { M) } 12 \%\end{array}$ & - & Free & $\mathrm{FOD}(6)$ \\
\hline 2003 & Doganay et al. [10] & NA & Few/+/- & G) $1 \%, M) 8 \%$ & - & Free & FOD(34) \\
\hline 2004 & Ru et al. [11] & G & A few/NA/- & $<5 \sim 20 \%$ & + & Free & $\mathrm{FOD}(8)$ \\
\hline 2007 & Chao et al. [12] & NA & $-/ N A / N A$ & 2.8 & + & Free & $\mathrm{FOD}(6)$ \\
\hline 2007 & Musulimani et al. [13] & NA & -/NA/NA & NA & NA & Free & $\begin{array}{l}\text { recurred bilateral lung lesions, } \\
\text { Tumor bearing(48) }\end{array}$ \\
\hline \multirow[t]{5}{*}{2007} & \multirow[t]{5}{*}{ Chang et al. [14] } & G & Rare/NA/NA & $<5 \%$ & NA & NA & $\mathrm{FOD}(31)$ \\
\hline & & G & Rare/NA/NA & $<5 \%$ & NA & NA & FOD(14) \\
\hline & & G & Rare/NA/NA & $<5 \%$ & NA & NA & FOD(13) \\
\hline & & G & Rare/NA/NA & $<5 \%$ & NA & NA & FOD(78) \\
\hline & & G & Rare/NA/NA & $<5 \%$ & NA & Bilateral lung nodules. & $\begin{array}{l}\text { No recurrence(5) not changed in } \\
\text { appearance on a follow-up CT }\end{array}$ \\
\hline \multirow[t]{5}{*}{2009} & \multirow[t]{5}{*}{ Nguen et al. [15] } & NA & \multirow{5}{*}{$\begin{array}{l}\text { Rare/NA/ } \\
1 \text { case: } L y(+), V(+), N(+)\end{array}$} & NA & NA & \multirow{5}{*}{$\begin{array}{l}\text { 1/5 case: infiltrated } \\
\text { peribronchial tissue and } \\
\text { LN metastasis. }\end{array}$} & $\mathrm{FOD}(4)$ \\
\hline & & NA & & NA & NA & & FOD(12) \\
\hline & & NA & & NA & NA & & NA \\
\hline & & NA & & NA & NA & & $\mathrm{FOD}(12)$ \\
\hline & & NA & & NA & NA & & $\mathrm{FOD}(4)$ \\
\hline 2009 & Rosenfeld et al. [16] & NA & Rare few/-/NA & NA & NA & $\begin{array}{l}\text { The biphasic neoplastic } \\
\text { cells replaced part of a } \\
\text { lymph node. }\end{array}$ & $\mathrm{FOD}(12)$ \\
\hline 2011 & Nishihara et al. [17] & NA & $(-/-/ N A)($ biopsy) & (10\%)(biopsy) & NA & NA/skull metastasis & NA \\
\hline 2011 & Munoz et al. [18] & G & $-1-1-$ & NA & NA & Free & NA \\
\hline 2011 & Kang et al. [19] & NA & NA & NA & NA & Free & $\begin{array}{l}\text { 1/2 case: recurrence; ipsilateral } \\
\text { lung, pneumonectomy }\end{array}$ \\
\hline
\end{tabular}


Table 2 Review of P-EMC cases: Cases and clinicopathological features (Continued)

\begin{tabular}{|c|c|c|c|c|c|c|c|}
\hline Year & Author & $\begin{array}{l}\text { Predominant } \\
\text { component }\end{array}$ & $\begin{array}{l}\text { High mitotic rate/ } \\
\text { necrosis/Ly,V,N invasion }\end{array}$ & Ki-67 & p53 & Metastasis & F/U (months) \\
\hline 2012 & Arif et al. [20] & $G$ & $(2-3 / 10 \mathrm{HPF}) /-/ \mathrm{NA}$ & $2-3 \%$ & NA & Free & $\mathrm{FOD}(9)$ \\
\hline 2013 & Zhu et al. [21] & NA & NA/NA/NA & NA & NA & Free & $\begin{array}{l}\text { 5-year OS, } 100 \% \text {, } \\
1 \text { case: mets to bone within } \\
3 \text { years, } \\
\text { Others: } \mathrm{FOD}(\sim 60)\end{array}$ \\
\hline 2013 & Konoglou et al. [22] & NA & NA/NA/NA & $\begin{array}{l}\text { Particularly } \\
\text { low }\end{array}$ & NA & - & $\mathrm{FOD}(24)$ \\
\hline 2014 & Cho et al. [23] & NA & A few/NA/NA & NA & NA & Free & $\mathrm{FOD}(16)$ \\
\hline \multirow[t]{5}{*}{2014} & \multirow[t]{5}{*}{ Song et al. [24] } & $M(>95 \%)$ & $-/-/ N A$ & NA & NA & Free & $\begin{array}{l}\text { Recurrence(33), } \\
\text { Complete pneumonectomy, } \\
\text { mets to chest wall(37), } \\
\text { Died of P-EMC(117) }\end{array}$ \\
\hline & & $M(30 \%)$ & $-/+/ N A$ & NA & NA & Free & $\mathrm{FOD}(75)$ \\
\hline & & $M(60 \%)$ & $-/-/ N A$ & NA & NA & Free & FOD(33) \\
\hline & & $M(70 \%)$ & $-/-/ N A$ & NA & NA & Free & $\mathrm{FOD}(1)$ \\
\hline & & $M(40 \%)$ & $-/-/ N A$ & NA & NA & Free & $\mathrm{FOD}(10)$ \\
\hline 2015 & Cha et al. [25] & M & $\mathrm{NA} /+/-$ & $\begin{array}{l}\text { G) }<1 \% \\
\text { M) } 40 \%\end{array}$ & NA & Hilar LN+subcarinal LN+ & Adj Chemo \\
\hline 2015 & Tajima et al. [26] & $M(70-90 \%)$ & A few/-N(+) & $\begin{array}{l}\text { G) } 1.6 \% \\
\text { M) } 2.8-14.2 \%\end{array}$ & $\begin{array}{l}\text { a } \\
\text { few } \\
+\end{array}$ & Free & $\mathrm{FOD}(4)$ \\
\hline 2016 & Shen et al. [27] & NA & NA/NA/NA & NA & NA & Free & $\mathrm{FOD}(8)$ \\
\hline Curre & nt case & G & Rare/+/- & $<5 \%$ & NA & Free & FOD(36) \\
\hline
\end{tabular}

$G$ dual layered glands, $M$ solid or sheets of myoepithelial cells, $N A$ not available, $M()$ percentage of the myoepithelial component, $L y$ lymphatic, $V$ vascular, $N$ neural, $H P F$ high-power field, LN lymph node, F/U Follow-up, FOD free of disease, mets metastasis, CRTx chemotherapy and radiotherapy, Adj Adjuvant

cases-wide edge resection (5 cases), excision by bronchoscopy (4 cases), and biopsy by bronchoscopy (1 case). Chao et al. performed bronchoscopic excision, because the patient refused a surgical procedure and the tumor growth was limited into the bronchial cartilage layer. The doctors argued that curative electrosurgery was an option for management of this low-grade malignancy [12]. In contrast, the case of Musulimani et al. revealed residual and/or recurrent P-EMC 8 months after their patient underwent a bronchoscopy that revealed a bilateral lung metastatic lesion; however, he remained asymptomatic and clinically healthy after 4 years [13]. Therefore, bronchoscopic resection could be a viable option, especially when passive treatment is desired. We think that it is necessary to explain sufficiently to the patient that additional surgical resection is needed in order to examine whether the residual tumor contains elements suggesting poor prognosis.

Among 56 cases we reviewed, 3 cases of metastatic lymph nodes were found at surgery. Moreover, only 7 reported the performance of systematic lymph node dissection. Although the necessity of lymph node dissection is unclear, sampling of lymph nodes to establish the cancer stage is considered a beneficial option, especially if there are any findings that suggest tumor aggressiveness. Therefore, we suggest that evaluation of lymph node metastasis provides valuable information in post-operative follow-up due to the unproven malignant potential of P-EMCs. In salivary gland EMCs, it has been reported that there are long intervals between original treatment and recurrence (mean, 5 years) or metastasis (mean, 15 years) [26]. In our review, there are six recurrent cases after the surgical treatment, and the interval was 8 months ( 1 case), 2 years (1 case), 3 years (3 cases), or data not available (1 case) [3, $13,19,21,24]$. These data indicate that a thorough follow-up of at least 3 years is necessary after surgery.

\section{Conclusions}

Here, we report a case of P-EMC for the rarity. Although the majority of P-EMCs behave indolently as seen in our case, our review indicates that several P-EMCs progress. Histological findings such as myoepithelial anaplasia could be a predictive factor for distinguishing the minor malignant cases from others. Complete resection is needed to evaluate the whole tumor, since P-EMC usually shows histological heterogeneity and since incomplete excision may be a poor prognostic factor. Until now, lobectomies, as well as lymph node dissections, sleeve lobectomies, or pneumonectomies, have been frequently performed for complete resection of P-EMC. Further investigation is required to establish the optimal treatment strategy. 


\section{Abbreviations}

CT: Computed tomography; DFS: Disease-free survival; EMC: Epithelialmyoepithelial carcinoma; FDG-PET: $F^{18}$-fluoro-deoxy-glucose positron emission tomography; HGT: High-grade transformation; MRI: Magnetic resonance imaging; P-EMC: Pulmonary epithelial-myoepithelial carcinoma; SMA: Smooth muscle actin

\section{Acknowledgements}

The authors would like to thank Editage (https://www.editage.jp) for the English language editing.

\section{Availability of data and materials}

The data supporting the conclusions of this article are included within the article.

\section{Authors' contributions}

YN participated in the conception, design, and analysis of this case report and drafted the manuscript. RM, AU, and $\mathrm{KI}$ participated in the design and coordination of the report and helped to draft the manuscript. TY was the chief supervisor. All authors read and approved the final manuscript.

\section{Authors' information}

$\mathrm{RM}$ is the director in the Department of Chest surgery, Tokyo Yamate Medical Center. Kl is the director in the Department of Pathology, Tokyo Yamate Medical Center. TY is a professor of the Department of Pathology, Dokkyo Medical University.

\section{Ethics approval and consent to participate}

Not applicable.

\section{Consent for publication}

The patient provided consent for the use of her personal data.

\section{Competing interests}

The authors declare that they have no competing interests.

\section{Publisher's Note}

Springer Nature remains neutral with regard to jurisdictional claims in published maps and institutional affiliations.

\section{Author details}

'Department of Chest Surgery, Tokyo Yamate Medical Center, 3-22-1 Hyakunin-cho, Shinjuku-ku, Tokyo 169-0073, Japan. ${ }^{2}$ Department of Pathology, Tokyo Yamate Medical Center, 3-22-1 Hyakunin-cho, Shinjuku-ku, Tokyo 169-0073, Japan. ${ }^{3}$ Department of Thoracic Surgery, Tokyo Medical and Dental University, 1-5-45, Yushima, Bunkyo-ku, Tokyo 113-8519, Japan. ${ }^{4}$ Department of Pathology, Dokkyo Medical University, 880 Kitakobayashi, Mibu-machi, Shimotsuga-gun, Tochigi 321-0293, Japan.

Received: 29 December 2017 Accepted: 2 July 2018

Published online: 09 July 2018

\section{References}

1. Ishikawa Y, Dacic S, Husain AN, Nicholson AG. Epithelial-myoepithelial carcinoma. In: Travis WD, Brambilla E, Burke AP, editors. World Health Organization classification of tumours of the lung, pleura, thymus and heart. 4th ed. Lyon: IARC Press; 2015. p. p103-4.

2. Pelosi G, Rodriguez J, Viale G, Rosai J. Salivary gland-type tumors with myoepithelial differentiation arising in pulmonary hamartoma: report of 2 cases of a hitherto unrecognized association. Am J Surg Pathol. 2006;30:375-87.

3. Moran CA, Suster S, Askin FB, Koss MN. Benign and malignant salivary gland-type mixed tumors of the lung. Clinicopathologic and immunohistochemical study of eight cases. Cancer. 1994;73:2481-90.

4. Nistal M, García-Viera M, Martínez-García C, Paniagua R. Epithelialmyoepithelial tumor of the bronchus. Am J Surg Pathol. 1994;18:421-5.

5. Tsuji N, Tateishi R, Ishiguro S, Terao T, Higashiyama M. Adenomyoepithelioma of the lung. Am J Surg Pathol. 1995;19:956-62.

6. Wilson RW, Moran CA. Epithelial-myoepithelial carcinoma of the lung: immunohistochemical and ultrastructural observations and review of the literature. Hum Pathol. 1997;28:631-5.
7. Shanks JH, Hasleton PS, Curry A, Rahman A. Bronchial epithelialmyoepithelial carcinoma. Histopathology. 1998;33:90-1.

8. Ryska A, Kerekes Z, Hovorková E, Barton P. Epithelial carcinoma of the bronchus. Pathol Res Pract. 1998;194:431-5.

9. Fulford LG, Kamata Y, Okudera K, Dawson A, Corrin B, Sheppard MN, et al. Epithelial-myoepithelial carcinomas of the bronchus. Am J Surg Pathol. 2001;25:1508-14.

10. Doganay L, Bilgi S, Ozdil A, Yoruk Y, Altaner S, Kutlu K. Epithelialmyoepithelial carcinoma of the lung. A case report and review of the literature. Arch Pathol Lab Med. 2003;127:177-80.

11. Ru K, Srivastava A, Tischler AS. Bronchial epithelial-myoepithelial carcinoma. Arch Pathol Lab Med. 2004;128:92-4.

12. Chao TY, Lin AS, Lie CH, Chung YH, Lin JW, Lin MC. Bronchial epithelialmyoepithelial carcinoma. Ann Thorac Surg. 2007 Feb;83:689-91.

13. Muslimani AA, Kundranda M, Jain S, Daw HA. Recurrent bronchial epithelialmyoepithelial carcinoma after local therapy. Clin Lung Cancer. 2007;8:386-8.

14. Chang T, Husain AN, Colby T, Taxy JB, Welch WR, Cheung OY, et al. Pneumocytic adenomyoepithelioma: a distinctive lung tumor with epithelial, myoepithelial, and pneumocytic differentiation. Am J Surg Pathol. 2007:31:562-8.

15. Nguyen CV, Suster S, Moran CA. Pulmonary epithelial-myoepithelial carcinoma: a clinicopathologic and immunohistochemical study of 5 cases. Hum Pathol. 2009;40:366-73.

16. Rosenfeld A, Schwartz D, Garzon S, Chaleff S. Epithelial-myoepithelial carcinoma of the lung: a case report and review of the literature. J Pediatr Hematol Oncol. 2009:31:206-8

17. Nishihara M, Takeda N, Tatsumi S, Kidoguchi K, Hayashi S, Sasayama T, et al. Skull metastasis as initial manifestation of pulmonary epithelialmyoepithelial carcinoma: a case report of an unusual case. Case Rep Oncol Med. 2011; https://doi.org/10.1155/2011/610383.

18. Muñoz G, Felipo F, Marquina I, Del Agua C. Epithelial-myoepithelial tumour of the lung: a case report referring to its molecular histogenesis. Diagn Pathol. 2011:6:71.

19. Kang DY, Yoon YS, Kim HK, Choi YS, Kim K, Shim YM, et al. Primary salivary gland-type lung cancer: surgical outcomes. Lung Cancer. 2011;72:250-4.

20. Arif F, Wu S, Andaz S, Fox S. Primary epithelial myoepithelial carcinoma of lung, reporting of a rare entity, its molecular histogenesis and review of the literature. Case Rep Pathol. 2012; https://doi.org/10.1155/2012/319434.

21. Zhu F, Liu Z, Hou Y, He D, Ge X, Bai C, et al. Primary salivary gland-type lung cancer: clinicopathological analysis of 88 cases from China. J Thorac Oncol. 2013;8:1578-84

22. Konoglou M, Cheva A, Zarogoulidis P, Porpodis K, Pataka A, Mpaliaka A, et al. Epithelial-myoepithelial carcinoma of the trachea-a rare entity case report. J Thorac Dis. 2014; https://doi.org/10.3978/j.issn.2072-1439.2013.11.17.

23. Cho SH, Park SD, Ko TY, Lee HY, Kim Jl. Primary epithelial myoepithelial lung carcinoma. Korean J Thorac Cardiovasc Surg. 2014:47:59-62.

24. Song DH, Choi IH, Ha SY, Han KM, Han J, Kim TS, et al. Epithelialmyoepthelial carcinoma of the tracheobronchial tree: the prognostic role of myoepithelial cells. Lung Cancer. 2014;83:416-9.

25. Cha YJ, Han J, Lee MJ, Lee KS, Kim H, Zo J. A rare case of bronchial epithelial-myoepithelial carcinoma with solid lobular growth in a 53-yearold woman. Tuberc Respir Dis. 2015;78:428-31.

26. Tajima S, Aki M, Yajima K, Takahashi T, Neyatani H, Koda K. Primary epithelial-myoepithelial carcinoma of the lung: a case report demonstrating high-grade transformation-like changes. Oncol Lett. 2015;10:175-81.

27. Shen $C$, Wang $X$, Che G. A rare case of primary peripheral epithelial myoepithelial carcinoma of lung: case report and literature review. Medicine. 2016;95:4371.

28. Seethala RR, Hunt JL, Baloch ZW, Livolsi VA, Leon Barnes E. Adenoid cystic carcinoma with high-grade transformation: a report of 11 cases and a review of the literature. Am J Surg Pathol. 2007:31:1683-94. 